\title{
Theory for the Photoacoustic Response to X-Ray Absorption
}

\author{
M. E. Garcia, G. M. Pastor, and K. H. Bennemann \\ Institute for Theoretical Physics, Freie Universität Berlin, D-1000 Berlin 33, West Germany \\ (Received 28 January 1988)
}

\begin{abstract}
A microscopic theory is presented for the photoacoustic effect induced in solids by $x$-ray absorption. The photoacoustic effect results from the thermalization of the excited Auger electrons and photoelectrons. We explain the dependence of the photoacoustic signal $S$ on photon energy and the proportionality to the $\mathrm{x}$-ray absorption coefficient in agreement with recent experiments on $\mathrm{Cu}$. Results are presented for the dependence of $S$ on photon energy, sample thickness, and the electronic structure of the absorbing solid.
\end{abstract}

PACS numbers: $78.20 . \mathrm{Hp}, 78.70 . \mathrm{Dm}$

The photoacoustic effect has become a subject of intensive research because of its fundamental importance and potential for numerous applications. ${ }^{1-5}$ So far, the photoacoustic response to optical radiation with wavelength $\lambda \cong 10^{3} \AA$ has been studied. ${ }^{1}$ Little attention is paid to the mechanisms of heat production and good agreement with experiment is obtained by the assumption that the amount of heat produced is a fraction of the absorbed energy and independent of the photon energy. However, as indicated by recent experiments ${ }^{2,3}$ (see Fig. 1 ), this is not the case for the photoacoustic signal resulting from $x$-ray absorption. In particular, note that the relative increases of the $\mathrm{x}$-ray absorption coefficient $\mu$ and of the photoacoustic signal $S$ at the $K$ edge are different. It is remarkable that in spite of the complex nonlocal electronic thermalization processes involved in the heat production within the solid, the oscillations ${ }^{6}$ of $\mu$ are imaged in $S$. It is the purpose of this Letter to present a microscopic theory for the photoacoustic response to $\mathrm{x}$-ray absorption in solids, which explains these new experimental facts like the observed dependence of $S$ on photon energy, sample thickness, and electronic structure of the absorbing solid $(\mathrm{Cu}, \mathrm{Al}, \mathrm{Pb}$, etc.). The theory presented here should provide a basis for a better understanding of the photoacoustic response and its dependence on the electronic structure of the solid, and should be useful for the use of photoacoustic x-ray absorption spectroscopy as a new experimental technique.

In order to find an expression for the photoacoustic signal $S$ which relates $S$ to microscopic processes in the solid, we use the following physical picture for the pho-

$$
s(\hbar \omega, x)=\left(c_{s} \rho_{s} d\right)^{-1} \sum_{i} w_{i}(\hbar \omega, x) \alpha_{i}(\hbar \omega, x) \epsilon_{i}(\hbar \omega),
$$

toacoustic response to $\mathrm{X}$-ray absorption. $\mathrm{X}$ rays are $\mathrm{ab}$ sorbed by the excitation of core electrons to states belonging to the continuum part of the electronic energy spectrum (photoelectrons). The excited electrons have a small mean free path, $l \cong 10-100 \AA$. The excited atom decays either by electromagnetic radiation, which may be reabsorbed, or by an Auger process. The resulting Auger electrons can have much larger energies than the photoelectrons. However, their mean free path is at most $100 \AA$ for elements with atomic number $Z \lesssim 40$. Then Auger electrons and photoelectrons transfer part of their kinetic energy to the solid, for example, by the production of cascades of secondary electrons. The first step in such processes consists of electron-electron interactions and is essentially nonlocal. After many collisions these electrons are close to thermal equilibrium. Note that during this thermalization, energy loss due to fluorescence or electron emission may occur. The second step in the transfer of energy from the system of thermalized excited electrons to the system of atoms of the solid is governed by the electron-phonon interaction bringing the electrons and the lattice to a common temperature. $^{7}$ Then by diffusion processes the transferred energy is distributed over the sample, increasing its initial temperature. Note that the transferred energy is distributed only within that part of the sample volume $V_{D}$ which is reached by diffusion within the $\mathrm{x}$-ray chopping period. Finally, the rise in temperature of the solid generates at the interface between the sample and the coupling gas the pressure waves which are detected as a photoacoustic signal. ${ }^{8}$ According to this physical picture the contribution to $S$ due to x-ray absorption at the point $x$ is given by

where $\hbar \omega$ is the energy of the incident $\mathrm{x}$ ray, $w_{i}$ is the probability for the $i$ th electron to be excited to a state with kinetic energy $\epsilon_{i}$, and $\alpha_{i}$ is the fraction of $\epsilon_{i}$ which is transformed into heat within the solid. $c_{s}$ refers to the specific heat, $\rho_{s}$ to the density of the absorbing solid, and $d$ to the sample thickness. The sum runs over all excited electrons. For samples much thicker than the electron mean free path, surface losses can be neglected and $\alpha_{i}$ is then independent of $x$. The total contribution to $S$ is obtained by integration of $s(x)$ over the volume of the absorbing solid, or more accurately over that part of the sample volume which contributes to the temperature increase at the surface. Thus it follows from

(C) 1988 The American Physical Society 
Eq. (1) that

$$
\begin{aligned}
S(\hbar \omega)=\left(c_{s} \rho_{s} d\right)^{-1}\left\{\sum_{i=K, L, M, \ldots}\left(1-e^{-\mu_{1} d}\right) \alpha_{i}(\right. & \left(\hbar \omega-\epsilon_{i}\right)+\sum_{\substack{i, j, k \\
\epsilon_{i}<\epsilon_{j}, \epsilon_{k}}}\left(1-e^{-\mu_{t} d}\right) \alpha_{i j k} p_{i j k}^{A} \epsilon_{i j k} \\
& \left.+\sum_{\substack{i, j, k \\
\epsilon_{i}<\epsilon_{j}, \epsilon_{k}}} \int d x d \Omega \mu_{i} e^{-\mu_{t} x} p_{i j}^{F}(\theta, \phi) P_{k}(x, \theta) \alpha_{i j k}\left(\epsilon_{i}-\epsilon_{j}-\epsilon_{k}\right)\right\} .
\end{aligned}
$$

Here, $i, j, k$ refer to shells of core electrons, whose energies are given with respect to the Fermi level. $\mu_{i}$ is the absorption coefficient referring to an electron in the $i$ th shell and $\epsilon_{i j k}=\epsilon_{i}-\epsilon_{j}-\epsilon_{k}$ refers to the energy of the electron excited by the $(i j k)$ Auger process. $p_{i j k}^{A}$ is the probability for the occurrence of the (ijk) Auger transition. $\alpha_{i}$ and $\alpha_{i j k}$ are functions of the excitation energy: $\alpha_{i}=\alpha\left(\hbar \omega-\epsilon_{i}\right), \alpha_{i j k}=\alpha\left(\epsilon_{i j k}\right)$. In deriving Eq. (2) we used the facts that $w_{i}(\hbar \omega, x)=\mu_{i} e^{-\mu_{i} x}$ for $i$-shell photoelectrons and $w_{i}(\hbar \omega, x)=\mu_{i} e^{-\mu_{i} x} p_{i j k}^{A}$ for $(i j k)$-Auger electrons. The first term in Eq. (2) gives the contribution to $S$ resulting from the photoelectrons. Note that this contribution arises even if the deexcitation of the electrons is produced only by fluorescence. The second term in Eq. (2) represents the contribution of the Auger electrons emitted from the shell $k$ due to transitions $i \rightarrow j$. Finally, the last term in Eq. (2) takes into account the partial reabsorption by electrons of the shell $k$ of the radiation caused by fluorescence resulting from transitions $i \rightarrow j$. The fluorescence probability for the shell $i$ (which, for simplicity, we assume to be isotropic) is denoted by $p_{i j}^{F}$ and the probability for this radiation to be absorbed by the shell $k$ by $P_{k}$. The kinetic energy of the resulting photoelectron from the $k$ th shell is given by $\epsilon_{i}-\epsilon_{j}-\epsilon_{k}$

Equation (2) is the central equation of our theory and is now used to explain important experimental facts. For this and numerical analysis we assume the following simplifications. We consider explicitly only the most relevant absorption channels. These are, below the $K$ edge, the $L$-shell absorption, and above the $K$ edge, the $L$ - and $K$-shell absorptions. We consider the average energies $\epsilon_{R}^{A}$ and $\epsilon_{L}^{A}$ of the $K$ and $L$ Auger electrons. Similarly, for $p_{i j k}^{A}$ we use $p_{K}^{A}$ and $p_{L}^{A}$, referring to the probabilities of emission of an Auger electron as a result of holes in the $K$ and $L$ shells, respectively. The values of these parameters are summarized in the figure captions. Since no experimental results are available for the energy dependence of $\alpha_{i}$ and since first-principles calculations seem at present very difficult, we use $\alpha_{i}$ as a parameter. Furthermore, on physical grounds one expects that electrons with similar excitation energies should have the same value of $\alpha$, since for all of them the same kind of processes are involved in thermalization. Therefore, we use $\alpha_{i}=\alpha_{1}$ for low-energy electrons like the $L$-shell Auger electrons and $K$-shell photoelectrons, and $\alpha_{i}=\alpha_{2}$ for the $K$-shell Auger electrons and $L$-shell photoelectrons. With these simplifications Eq. (2) is rewritten as

$$
\begin{aligned}
S(\hbar \omega)=\left(c_{s} \rho_{s} d\right)^{-1}\left\{\left(1-e^{-\mu_{K} d}\right) \alpha_{1}\left(\hbar \omega-\epsilon_{K}\right)\right. & +\left(1-e^{-\mu_{L} d}\right) \alpha_{2}\left(\hbar \omega-\epsilon_{L}\right)+\left(1-e^{-\mu_{K} d}\right) \alpha_{2} p_{K}^{A} \epsilon_{K}^{A} \\
& \left.+\left(1-e^{-\mu_{L} d}\right) \alpha_{1} p_{L}^{A} \epsilon_{L}^{A}+\int d x d \Omega \mu_{K} e^{-\mu_{K} x} p_{K}^{F} \alpha_{2} P_{L}(x, \theta)\left(\epsilon_{K}-2 \epsilon_{L}\right)\right\} .
\end{aligned}
$$

Here, we assume that radiation results from $L \rightarrow K$ transitions and is absorbed by $L$-shell electrons. Note that the first term in Eq. (3) contributes only for energies above the $K$ edge; similarly the second term results only above the $L$ edge (formally, we set $\mu_{K}=0$ for $\hbar \omega<\epsilon_{K}$ and $\mu_{L}=0$ for $\left.\hbar \omega<\epsilon_{L}\right)$. For simplicity we assume $\mu_{\text {expt }}=\mu_{L}$ for $\hbar \omega<\epsilon_{K}$ since the excitation cross section for higher shells is negligible at these frequencies. For $\hbar \omega>\epsilon_{K}$ we use the same value for $\mu_{L}$ as below the $K$ edge and $\mu_{K}=\mu_{\text {expt }}-\mu_{L}$. Here, $\mu_{\text {expt }}$ refers to the experimentally observed absorption coefficient. It follows from Eqs. (2) and (3) that the photoacoustic response should increase with the photon energy $\hbar \omega$. Furthermore, because of $\mu_{K}(\hbar \omega)$, Eq. (3) yields that the photoacoustic signal $S$ exhibits the same structural dependence on photon energy as is observed in $\mathrm{x}$-ray absor- bance. It follows also from Eq. (3) that the sharp rise in $S$ at the $K$-shell edge is mainly determined by the thermalization of Auger electrons. Therefore, this increase of $S$ at the $K$ edge should exhibit a characteristic dependence on nuclear charge $(Z)$ due to $p_{K}^{A}(Z)$ for not too large values of $\hbar \omega-\epsilon_{K}$. The third and fourth terms in Eq. (3) give the dominant contribution to $S$ and depend on $\hbar \omega$ only via $\mu_{K}(\hbar \omega)$ and $\mu_{L}(\hbar \omega)$. Consequently, the local environment effects contained in $\mu_{K}(\hbar \omega)$ (for photon wavelength $\lambda<a, a=$ interatomic distance) are present in $S$ despite the fact that $S$ involves nonlocal thermalization processes. The structural dependence on $\hbar \omega$, like in x-ray absorption, is also preserved for the first term in Eq. (3) [via $\mu_{K}(\hbar \omega)$ ], since $\alpha$ is expected to exhibit no particular energy dependence. Note 
that $\hbar \omega-\epsilon_{i}$ is typically very large compared to the energies involved in electron-lattice coupling. Equation (3) also describes the dependence of $S$ on sample thickness for given $\hbar \omega$. Because of $c_{s}, \rho_{s}, \epsilon_{i}$, and $\mu_{i}$ different results are expected for $S(d)$ for $\mathrm{Cu}, \mathrm{Sn}, \mathrm{Ni}$, for example. These conclusions illustrate that Eqs. (2) and (3) are able to explain the essential experimental facts observed recently for the $x$-ray absorption-induced photoacoustic effect.

To demonstrate that our theory is also able to explain quantitatively experimental results, we show in Fig. 1 results for $S$ obtained from Eq. (3) with use of experimental values for $\mu_{L}$ and $\mu_{K},{ }^{2,9}$ semiempirical values for $\epsilon_{K}$, $\epsilon_{L}, \epsilon_{K}^{A}$, and $\epsilon_{L}^{A},{ }^{10}$ and experimental values for $p_{L}^{A}, p_{K}^{A}$, and $p_{K}^{F} .{ }^{11} P_{L}$ is given by $P_{L}=1-\exp \left[u_{L}(x-d) / \cos \theta\right]$ for $0<\theta<\pi / 2$ and $P_{L}=1-\exp \left(x \mu_{L} / \cos \theta\right)$ for $\pi / 2<\theta<\pi$. Results for $\alpha_{1}=\alpha_{2}$ and $\alpha_{1}=5 \alpha_{2}$ are given. Our theory is in fairly good agreement with experiment. The sharp rise in $S$ at the $K$ edge and the position and magnitude of the peaks of the oscillations in $S$ are correctly reproduced. Note that while experimentally $S$ increases for increasing $\hbar \omega$, the $\mathrm{x}$-ray absorbance decreases with $\hbar \omega$ above the edge. On general physical grounds one expects $\alpha_{1}>\alpha_{2}$, since the larger the excitation energy of the electron, the less its efficiency to produce heat because of the radiative losses in its collisions. Taking into account the energy loss due to fluorescence, we estimate $\alpha_{1} / \alpha_{2}$ to be at most of the order of 2 . Therefore, the value $\alpha_{1} / \alpha_{2}=5$

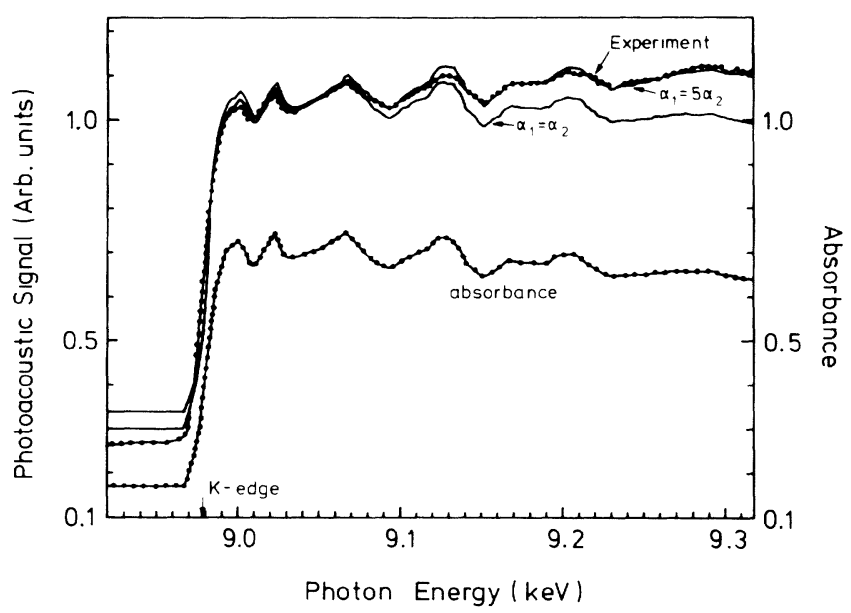

FIG. 1. X-ray-induced photoacoustic response signal $S$ as a function of photon energy $(\hbar \omega)$ for a $\mathrm{Cu}$ sample with thickness $d=5 \mu \mathrm{m}$ and $\mathrm{x}$-ray chopping frequency $10 \mathrm{~Hz}$. The dotted curve refers to experiment (Ref. 2) and the full curves to theory [Eq. (3)]. The x-ray absorbance $1-\exp \left(-\mu_{\exp } d\right)$ for the same sample is also shown. Note that the relative change in $S$ at the $K$ edge (which depends on the Auger decay probability and Auger-electron energy) is smaller than the corresponding change in the absorbance. The parameters $\mu_{L}, \mu_{K}$, $p_{L}^{A}, p_{K}^{A}, p F, \epsilon_{K}, \epsilon_{R}^{A}, \epsilon_{L}$, and $\epsilon_{L}^{A}$ are determined from experiment as discussed in the text $\left(p_{K}^{A}=0.61, p_{L}^{A}=0.98, \epsilon_{K}=8.98 \mathrm{keV}\right.$, $\epsilon_{K}^{A}=7.42 \mathrm{keV}, \epsilon_{L}=0.99 \mathrm{keV}, \epsilon_{L}^{A}=0.74 \mathrm{keV}$, and $\left.p{ }_{K}^{F}=1-p_{K}^{A}\right)$. seems somewhat too large. Photoacoustic response experiments on $\mathrm{Cu}$ performed with an electron beam seem to confirm a smooth decrease of $\alpha$ with increasing electron energy. ${ }^{12}$ The results obtained for $\alpha_{1}=\alpha_{2}$, which seems physically more realistic, and for $\alpha_{1}=5 \alpha_{2}$ illustrate how sensitively $S$ depends on the parameter $\alpha_{1} / \alpha_{2}$. Note that for $\alpha_{1}=\alpha_{2}$, the theoretical curve for $S$ exhibits the same structure as is experimentally observed. However, the increasing kinetic energy of the photoelectrons cannot overcome the decrease of the absorbance $1-\exp \left(-\mu_{K} d\right)$, see Eq. (3), and thus $S$ starts to decrease for energies about $100 \mathrm{eV}$ above the $K$ edge. Despite this discrepancy with experimental results, it seems reasonable to assume $\alpha_{1} \approx \alpha_{2}$, since the energetic electrons are only capable of exciting $L$-shell electrons, which have a fluorescence yield of only $1.1 \%$. Possibly the positive slope of the experimental curve for $S$ could be due to other experimental factors. This might also be the origin of the large rise of $S$ observed $^{2}$ at much higher energies $\hbar \omega$ for which the absorption coefficient $\mu$ has already decreased to one tenth of its magnitude at the $K$

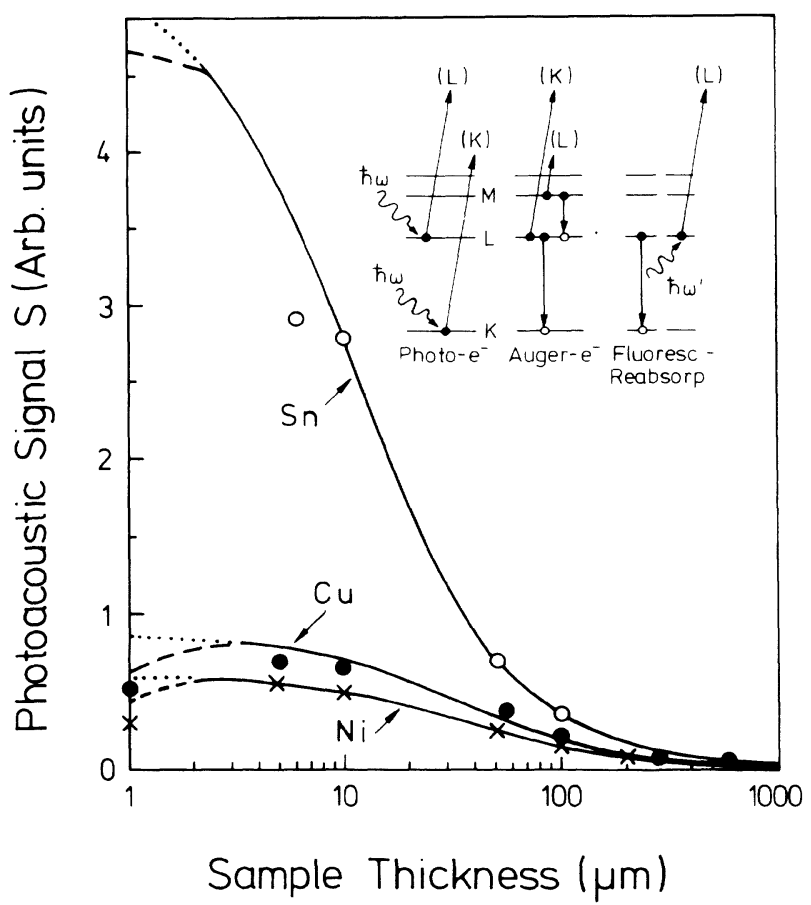

FIG. 2. Photoacoustic signal $S$ of $\mathrm{Cu}, \mathrm{Ni}$, and $\mathrm{Sn}$ with photon wavelength $\lambda=1.56 \AA$ and $x$-ray chopping frequency $10 \mathrm{~Hz}$ as functions of sample thickness $d$. Filled circles, crosses, and open circles refer to experimental results (Ref. 3) for $\mathrm{Cu}, \mathrm{Ni}$, and $\mathrm{Sn}$, respectively. Full curves refer to theory [Eq. (3)]. We use for $\mathrm{Cu}, \mathrm{Ni}$, and $\mathrm{Sn}$ the same value for $\alpha$. For small sample thickness we expect a change of the calculated $S$ (dotted curve) to smaller values (indicated qualitatively by the dashed curve) due to surface energy losses. Inset: Main processes which contribute to $S$, namely photoelectrons, Auger electrons, and reabsorbed radiation. 
edge. Presently, this large increase of $S$ for $\hbar \omega \gg \epsilon_{K}$ is not understood. Maybe it results from experimental uncertainties. $^{2}$ The photoacoustic signal $S$ below the $K$ edge is due to $L$-shell photoelectrons and Auger electrons. The contribution to $S$ due to the last terms of Eqs. (2) and (3) is small (smaller than $10 \%$ of the value of $S$ above the edge). Thus, the jump in $S$ is mainly due to the Auger electrons. ${ }^{13}$

In Fig. 2 results are given for the experimental and theoretical photoacoustic signals of $\mathrm{Ni}, \mathrm{Cu}$, and $\mathrm{Sn}$ as functions of sample thickness $d$. Only $\alpha$ is used as a fitting parameter for calculating $S(d)$ from Eq. (3). It is difficult to estimate the dependence of $\alpha$ on atomic number; however, we expect $\alpha$ to have similar values for different metals. Therefore, we used the same values for $\alpha$ for all of them. The agreement with experiment ${ }^{3}$ is quite good. For very small sample thickness the loss of photoexcited electrons at the surface reduces considerably the total $S$ and causes $S$ to decrease rapidly for $d \rightarrow 0$. For $d \simeq \mu^{-1}, S$ varies slowly with $d$ since the increase in the absorbance $1-e^{-\mu d}$ is somewhat compensated by the increase of the volume $V_{D}$ where the energy is distributed $\left(V_{D} \propto d\right)$. For $d$ much larger than the absorption length $\mu^{-1}$ but smaller than the diffusion length $\lambda_{s}\left(\mu^{-1} \gg d \gg \lambda_{s}\right)$, we obtain approximately $S \propto d^{-1}$ since further increase of $d$ causes no appreciable increase in the absorbance but a decrease in the temperature rise at the surface because the absorbed energy is distributed over a larger $V_{D}$. Although the trends for the studied materials $[S(\mathrm{Ni})<S(\mathrm{Cu})<S(\mathrm{Sn})<S(\mathrm{~Pb})]$ are already given by the difference in the absorbed energy $E_{\text {abs, }}$, the material-dependent prefactor $\left(c_{s} \rho_{s}\right)^{-1}$, which relates $E_{\text {abs }}$ to the temperature rise in the sample, ${ }^{1} \mathrm{im}$ proves quantitative agreement with experiment. Furthermore, assuming $\alpha_{\mathrm{Cu}}=\alpha_{\mathrm{Al}}=\alpha \mathrm{Pb}$, and for photon wavelength $\lambda=1.56 \AA, d_{\mathrm{Al}}=100 \mu_{\mathrm{m}}, d_{\mathrm{Cu}}=50 \mu_{\mathrm{m}}$, and $d_{\mathrm{Pb}}=50 \mu_{\mathrm{m}}$, we obtain $S_{\mathrm{Cu}} / S_{\mathrm{Al}}=1.7$ and $S_{\mathrm{Cu}} / S_{\mathrm{Pb}}=2.6$, while experiment yields for these ratios the values $2.0 \pm 0.6$ and 2.5 , respectively. It is remarkable that we obtain such agreement with the experimental results. ${ }^{2,3}$ In Fig. 2 we also illustrate the dependence of $S$ on the material and the importance of considering samples with the same thickness when comparing different absorbing solids.

We should remark that atomic motion of singly or doubly charged atoms resulting from photoexcitation of core electrons should play no significant role in the heat production, since the lifetime $\tau$ of the localized $K$ - and $L$-shell holes is much smaller $\left(\tau<10^{-15} \mathrm{~s}\right)$ than phonon oscillation periods. A contribution to $S$ due to direct creation of phonons resulting from the local charge unbalance caused by the core-electron emission cannot be completely disregarded, particularly when the holes have larger lifetimes (as may be the case for insulators).

In summary, we have presented a theory allowing a microscopic determination of the photoacoustic response to $x$-ray absorption. It is shown that the dependence of the photoacoustic signal on photon energy exhibits the same structure as in the case of $x$-ray absorbance.

We thank Professor T. Masujima et al. for communicating their results prior to publication, and M. Reichling and Professor E. Matthias for helpful discussions.

'A. C. Tam, Rev. Mod. Phys. 58, 381 (1986), and references therein.

${ }^{2}$ T. Masujima, H. Kawata, Y. Amemiya, N. Kamiya, T. Katsura, T. Iwamoto, H. Yoshida, H. Imai, and M. Ando, Chem. Lett. 1987, 973; T. Masujima, in Proceedings of the Fifth International Topical Meeting on Photoacoustic and Photothermal Phenomena, Heidelberg, West Germany, 1987, edited by P. Hess and J. Pelzl, Springer-Series in Optical Sciences Vol. 58 (Springer-Verlag, Heidelberg, 1988), pp. 19-24.

${ }^{3}$ T. Masujima, in "Topics in Current Chemistry," edited by E. Mandelkow (Springer-Verlag, Berlin, to be published), Vol. 2.

${ }^{4}$ A. Rosencwaig and A. Gersho, J. Appl. Phys. 47, 64 (1976).

${ }^{5}$ F. A. McDonald and G. C. Wetsel, Jr., J. Appl. Phys. 49, 2313 (1978).

${ }^{6}$ E. A. Stern, Phys. Rev. B 10, 3027 (1974).

${ }^{7}$ P. B. Allen, Phys. Rev. Lett. 59, 1460 (1987).

${ }^{8}$ In order to obtain the photoacoustic signal, one has to solve coupled linear equations for the time-dependent pressure and temperature in the gas (see Ref. 5). In our calculations we make the assumptions of the "thermal piston" approximation (see Ref. 4). The transfer of the heat produced in the solid to the gas (and its conversion to a photoacoustic signal) is taken into account by a constant transmission coefficient.

${ }^{9}$ International Tables for X-Ray Crystallography, Vol. III, edited by C. H. Macgillavry, G. D. Reick, and K. Lonsdale (Kynoch, Birmingham, England, 1962), pp. 157-192.

${ }^{10}$ F. P. Larkins, At. Data Nucl. Data Tables 20, 311 (1977).

${ }^{11}$ M. O. Krause, J. Phys. Chem. Ref. Data 8, 307 (1979).

${ }^{12}$ M. Reichling and Matthias, unpublished results.

${ }^{13} \mathrm{This}$ jump is characterized by the quantity $\delta=\left[S\left(E_{K}^{+}\right)\right.$ $\left.-S\left(E_{K}^{-}\right)\right] / S\left(E_{K^{-}}\right)$. Here, $E_{K^{+}}\left(E_{K^{-}}\right)$refers to the energy just above (below) the $K$ edge. It follows from Eq. (3) that

$$
\delta \simeq \frac{P_{K}^{A}\left[1-\exp \left(-\mu_{K} d\right)\right] \epsilon_{K}^{A}}{\left[1-\exp \left(-\mu_{L} d\right)\right]\left(\epsilon_{K}-\epsilon_{L}\right)}
$$

Here, we have for simplicity neglected small fluorescence contributions and $L$-shell Auger electrons. Note that $\delta$ is approximately independent of the fitting parameter $\alpha$ and depends on $P_{K}^{A}$, which is known to decrease for increasing atomic number, and on binding energies $\epsilon_{i}$. Thus, the quantity $\delta$ provides a direct experimental check of our theory. 